\title{
Molecular phenotyping of infiltrative cardiomyopathies: The future
}

\author{
Sharmila Dorbala, MD, MPH, ,a,b,c and Leslee J. Shaw, $\mathrm{PhD}^{\mathrm{d}}$ \\ a Division of Nuclear Medicine and Department of Radiology, Brigham and Women's Hospital, \\ Boston, MA \\ b Cardiac Amyloidosis Program, Heart and Vascular Center, Cardiovascular Division, Department \\ of Medicine, Brigham and Women's Hospital, Boston, MA \\ c Division of Nuclear Medicine and Department of Radiology, Brigham and Women's Hospital \\ and Harvard Medical School, Boston, MA \\ d Department of Cardiology, Cornell University Medical Center, New York
}

Received Nov 14, 2018; accepted Nov 14, 2018

doi: $10.1007 / \mathrm{s} 12350-018-01550-6$

\begin{abstract}
Cardiac sarcoidosis and amyloidosis have gained recent attention due to substantial advances in imaging and management. In this issue of the Journal of Nuclear Cardiology, imaging experts discuss the role of microvascular perfusion, innervation, targeted imaging of the heart and whole body, and novel molecular targets for imaging inflammation, fibrosis, and amyloidosis. In addition to cutting edge science, experts provide, for the first time, a patient page with information for patients with sarcoidosis and amyloidosis.
\end{abstract}

\section{Key Words: Cardiomyopathy • amyloid heart disease $\bullet$ sarcoid heart disease $\bullet$ PET • SPECT • infiltrative}

Infiltrative heart diseases exhibit substantial variation in pathogenesis, clinical manifestations, and clinical outcomes. ${ }^{1-3}$ Cardiac sarcoidosis and amyloidosis are the two infiltrative cardiomyopathies that have garnered most of the recent attention due to advances in imaging and management.

Advanced cardiovascular imaging has transformed the evaluation, management, and risk assessment of infiltrative heart diseases. Echocardiography is widely available and one of the most mature techniques with over 50 years of experience, but it detects advanced infiltration with increased left ventricular wall thickness/mass and is insensitive to diagnose early changes and response to therapy. Cardiac magnetic resonance

Funding Dr. Dorbala is supported by NIH RO1 Grant (RO1 HL 130563) and American Heart Association Grant (AHA 16 CSA 2888 0004).

Reprint requests: Sharmila Dorbala, MD, MPH, Cardiac Amyloidosis Program, Heart and Vascular Center, Cardiovascular Division, Department of Medicine, Brigham and Women's Hospital, Boston, MA, 2115; sdorbala@bwh.harvard.edu, sdorbala@partners.org J Nucl Cardiol 2019;26:154-7.

$1071-3581 / \$ 34.00$

Copyright (c) 2018 American Society of Nuclear Cardiology. imaging (CMR) is becoming increasingly available over the past decade, and provides high resolution, quantitative imaging with precise tissue characterization (fibrosis, inflammation edema, fat, and blood) and distinct phenotypic appearances (amyloidosis, sarcoidosis, Danon's disease, Fabry's disease). CMR, however, is not widely available, requires high technical expertise, and may be contraindicated in certain patients (eGFR $<30 \mathrm{~mL} / \mathrm{min}$, certain implanted devices etc.). Radionuclide imaging on the other hand is one of the most mature imaging techniques that is quantitative and provides exquisite sensitivity to detect pico/nano molar changes in the tissue. Moreover, radionuclide imaging offers distinct advantages over biopsy in characterizing infiltrative cardiomyopathy.

With the growth of molecularly targeted tracers to precisely phenotype infiltration, radionuclide imaging is likely to play an increasingly important role in imaging of infiltrative heart diseases. Tomographic radionuclide imaging offers significant advantages compared to histological diagnosis of infiltrative heart diseases such as sarcoidosis and amyloidosis (Figure 1). It provides whole organ, whole-body imaging, which can be used as a screening test in suspected infiltrative diseases, can be 

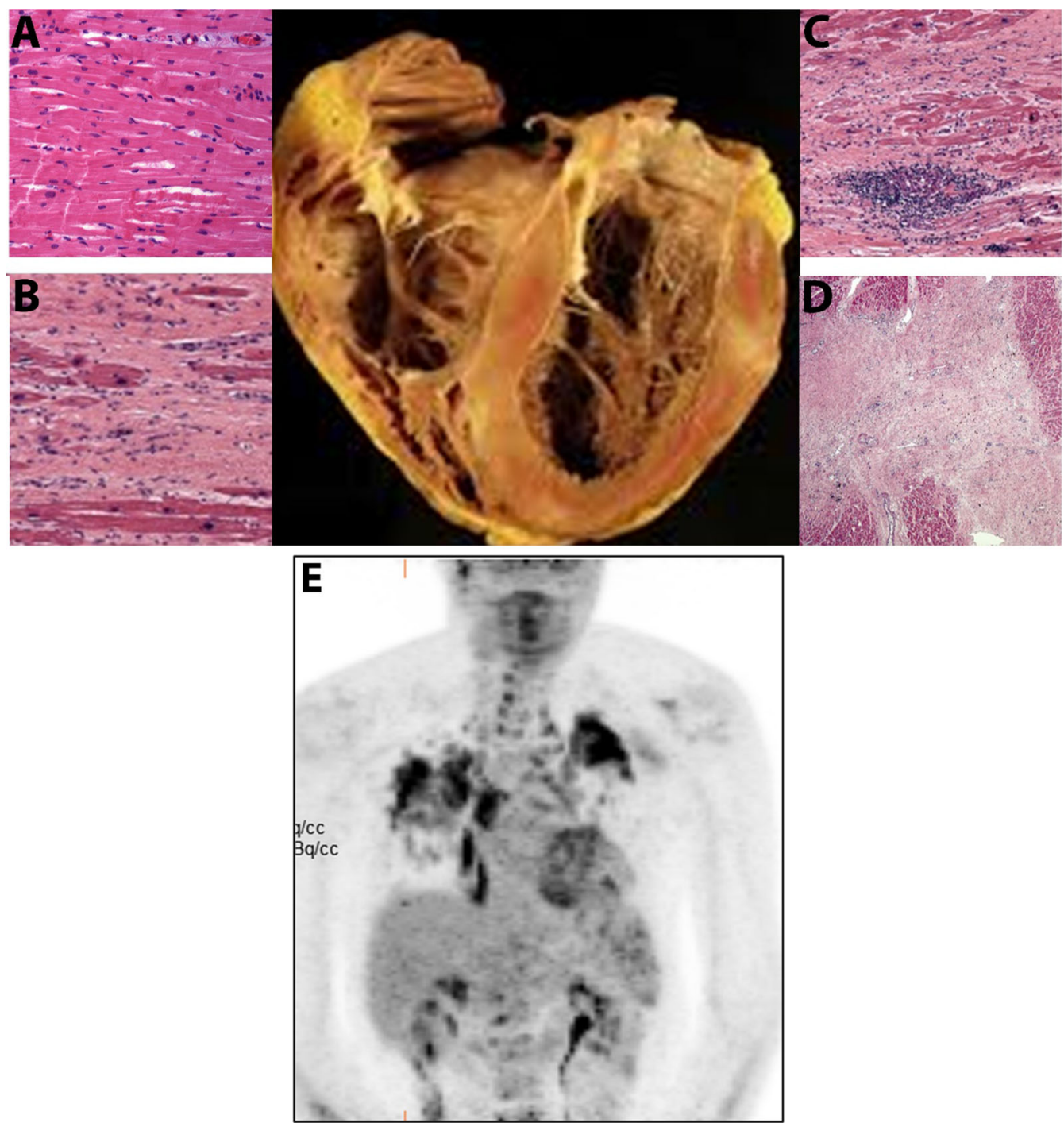

Figure 1. Advantages of imaging over endomyocardial biopsy in sarcoidosis. This figure shows a section of an explanted heart from a patient with advanced cardiac sarcoidosis who underwent cardiac transplantation. A blind endomyocardial biopsy from the right side of the interventricular septum may reveal, normal myocardium, non-specific inflammation $(\mathbf{A}, \mathbf{B})$, typical non-caseating granuloma from sarcoidosis $(\mathbf{C})$, or myocardial fibrosis (D). In sarcoidosis, these pathologies may coexist in different parts of the heart making imaging challenging to make management decisions based on random endomyocardial biopsy results. In contrast, imaging with ${ }^{18} \mathrm{~F}$-FDG PET/CT offers the ability to evaluate whole heart and whole-body burden of inflammation $(\mathbf{E})$.

quantified, repeated, and used to assess early disease response to therapy and to guide new drug development. In sarcoidosis, a blind endomyocardial biopsy from the right ventricular aspect of the septum may be limited, as sarcoid granulomas from the rest of the heart may be missed. An endomyocardial biopsy showing fibrosis or inflammation in the septum does not exclude extensive fibrosis or inflammation in the remaining myocardial segments. As therapy is guided by the presence of active inflammation/infiltration, imaging provides significant advantages. Moreover, imaging can guide the site of biopsy. The current paradigm of management of infiltrative heart diseases includes clinical evaluation combined with biopsy and imaging.

The pathogenesis of infiltrative cardiomyopathies varies widely. The myocytes and the extracellular matrix may change in structure (hypertrophy, atrophy, apoptosis, fibrosis, or a combination) and composition (edema, amyloid fibril deposition), and adapt to infiltrative pathology with complex metabolic switches (glucose, 


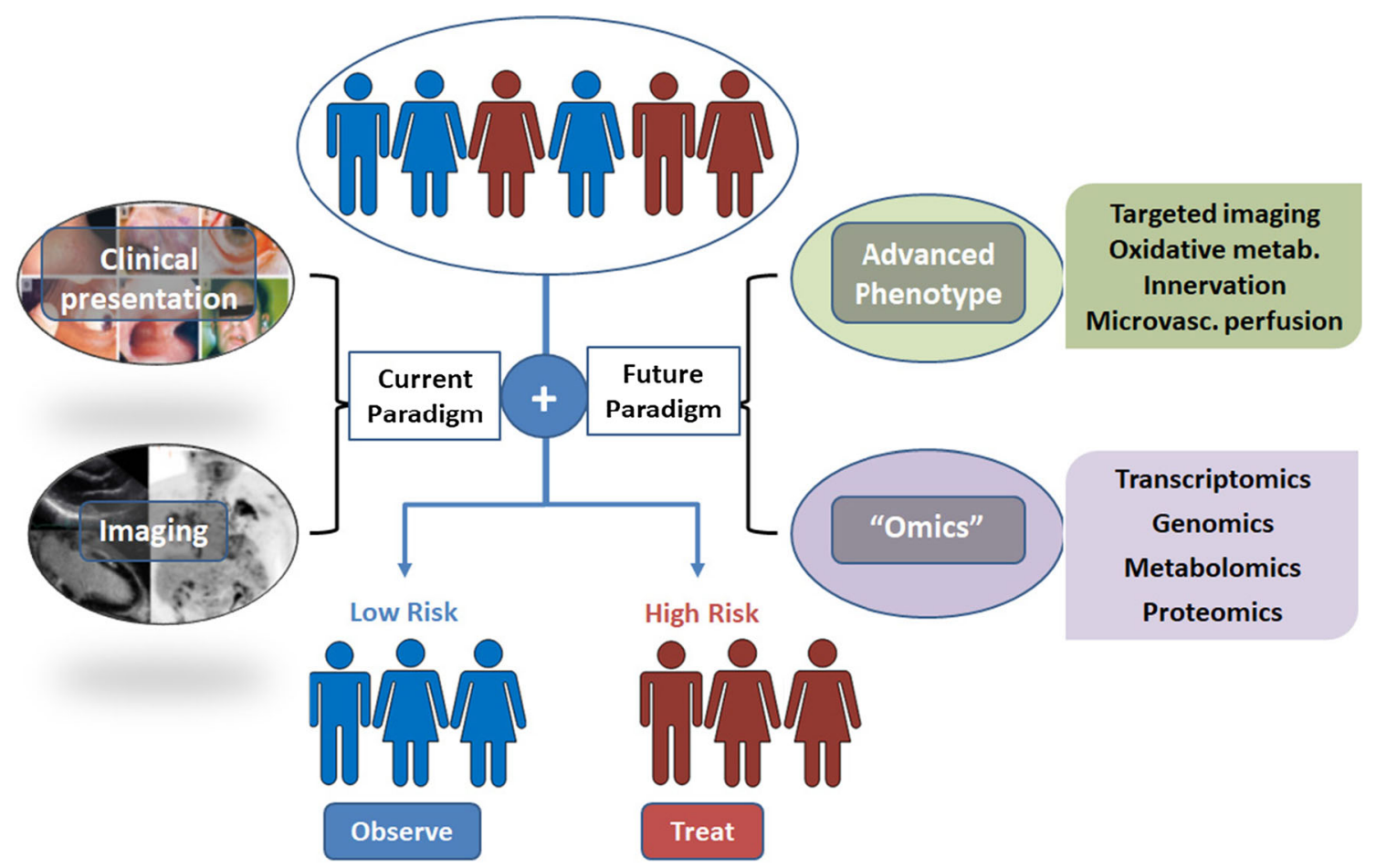

Figure 2. The future evaluation and management of infiltrative heart diseases: A combined approach including imaging, biopsy, and "omics." The current management of infiltrative heart diseases is based on the clinical presentation and imaging features, and biopsy when available. Personalized management of infiltrative heart diseases which also includes better characterization of cardiomyocyte adaptation and omics information is likely to improve outcomes. Parts of the figure are reproduced with permission from Iannuzzi et $\mathrm{al}^{1}$.

fatty acid, and oxidative metabolism). These structural and metabolic changes in the ventricles and atria can form substrates for electrical heterogeneity, cardiac rhythm abnormalities, heart blocks, and embolic phenomenon. ${ }^{2}$ Unfettered infiltration culminates in heart failure, morbidity, and death. A number of the myocardial adaptations to infiltration can be imaged non-invasively without a need for endomyocardial biopsy. Advanced phenotyping of myocardial metabolic processes and molecular adaptations evaluated by "omics" are likely to become the basis for personalized management of patients with infiltrative heart disease (Figure 2).

For the first time, effective targeted therapies ${ }^{4-6}$ will soon be available to slow progression of cardiac amyloidosis. ${ }^{6}$ In this issue of the Journal of Nuclear Cardiology, experts in the field discuss several topics including, emerging imaging targets for inflammation and fibrosis and the role of imaging in the evaluation of microvascular dysfunction, innervation, and targeted imaging of sarcoidosis and amyloidosis. In addition to cutting edge science, experts provide, for the first time, a patient page with information for patients with sarcoidosis and amyloidosis. Enjoy reading this theme issue of the Journal of Nuclear Cardiology on infiltrative cardiomyopathy.

\section{Disclosures}

Dr. Dorbala served as a consultant with Advanced Accelerator Applications, General Electric and Proclara. Dr. Shaw reports no relevant disclosures.

\section{References}

1. Iannuzzi MC, Rybicki BA, Teirstein AS. Sarcoidosis. N Engl J Med 2007;357:2153-65.

2. Falk RH. Diagnosis and management of the cardiac amyloidoses. Circulation 2005;112:2047-60.

3. Felker GM, Thompson RE, Hare JM, Hruban RH, Clemetson DE, Howard DL, et al. Underlying causes and long-term survival in patients with initially unexplained cardiomyopathy. N Engl J Med 2000;342:1077-84. 
4. Benson MD, Waddington-Cruz M, Berk JL, Polydefkis M, Dyck PJ, Wang AK, et al. Inotersen treatment for patients with hereditary transthyretin amyloidosis. N Engl J Med 2018;379:22-31.

5. Adams D, Gonzalez-Duarte A, O'Riordan WD, Yang CC, Ueda M, Kristen AV, Tournev I, Schmidt HH, Coelho T, Berk JL, Lin KP. Patisiran, an RNAi therapeutic, for hereditary transthyretin amyloidosis. N Engl J Med 2018;379:11-21.
6. Maurer MS, Schwartz JH, Gundapaneni B, Elliott PM, Merlini G, Waddington-Cruz $\mathrm{M}$, et al. Tafamidis treatment for patients with transthyretin amyloid cardiomyopathy. N Engl J Med 2018;379: 1007-16. 